\title{
Rehabilitation of Autism Spectrum Disorder (ASD) Children Using (RRR) Delta-3 Robot
}

\author{
S. Ayad, M. Ayad, and A. Megueni
}

\begin{abstract}
Our work concerns the development of the Delta-3 (RRR) parallel robot for the rehabilitation of autism spectrum disorder (ASD) children. The basic idea is to introduce training modes (passive and active) to the Delta-3 robot in order to help these children to stimulate their brains. In this paper the technical side (kinematic, dynamic and Control system) of rehabilitative Delta-3 robot is presented.

The kinematics study is used to calculate the forward and inverse kinematic and also velocities and accelerations for robot. The dynamic study allows to calculate the contribution of torque/forces acting on three motors to actuate the three arms of Delta- 3 robot and the Control system study is used to determine the necessary control elements for the motion of the robot. The Delta-robot's simulation result of a defined trajectory using SimMechanics and Simulink shows that our system has tracked the path, with a good spatial and temporal precision.
\end{abstract}

Index Terms - Delta-3 robot, biomechanics, rehabilitation.

\section{INTRODUCTION}

According to the Prevalence of Autism 2014 survey from the Centers for Disease Control and Prevention (CDC), 1 in 68 children in United States are identified having autism spectrum disorder (ASD) [1]. One of the effective therapeutic ways that is followed to serve the great number of autism children is using physical and mental devices, since ASD children tend to interact with technical devices more than with humans around them [2]. Thereby our work is looking for introducing the Delta-3 robot on the rehabilitation of ASD children as one of technological therapy solutions. Delta robot is one of the parallel robot types. It was invented during the 1980s by Reymond Clavel [3]. It is characterized by its lightness, precision and high acceleration that can exceed $50 \mathrm{~g}$. As a structural standpoint this robot is easy to assemble and disassemble. Since this robot requires little maintenance and is very flexible, hence you just need to change its end effector to reach a wide range of actions [4].The application field of Delta robot is more commonly known in the industry especially in the packaging.

According to the advantages that were sought above, the Delta-3 robot seems to be the convenient choice for helping ASD children to challenge their brains. Performing handicraft,

S. Ayad is with the Laboratory of Solids and Structures Mechanics, Faculty of Technology, University of Sidi-Bel Abbes, 22000, Algeria.

M. Ayad is with the Automatic laboratory, Faculty of Technology, University of Tlemcen, 13000, Algeria.

M. Megueni is with the Laboratory of Solids and Structures Mechanics, Faculty of Technology, University of Sidi-Bel Abbes, 22000, Algeria. solving puzzle games...etc. are some of the examples where Delta-3 robot can be useful. The motion assistance of the robot is provided in two different training modes. In the active mode, the robot performs most of the task in order to stimulate or train the child's brain. While in the passive mode, the task of the robot is to guide and intervene if need only.

This paper merely concerns the technical side of Delta-3 rehabilitation robot, hence the training modes and the medical study will not be encompassed. The paper is organized as follows: First, we have introduced the mechanical structure and the solutions of the kinematic and dynamic equations governing the behavior. Then we have studied the control and the stability of the system. Finally, we have realized modeled and simulated the Delta-3 robot and showed some results.

\section{ARCHITECTURE DESCRIPTION}

The Delta-3 robot as illustrated in Fig. 1 consists of two parallel platforms, the upper is fixed and named base platform, the lower is free to move in the three directions $\mathrm{x}, \mathrm{y}$ and $\mathrm{z}$ and called mobile platform. The movement of the mobile platform is generated by three rotational motors at the base platform, and transmitted through three parallel kinematic chains. Each chain consists of two serial arms:

- Upper arm: It has one rotational DOF, and is consisted of a bar that one of the extremities is connected with the actuator and the other with low arm.

- Lower arm (forearm): It has three rotational DOF, and is conceited of two parallel bars that are connected with the upper arm and the base platform via spherical joints.

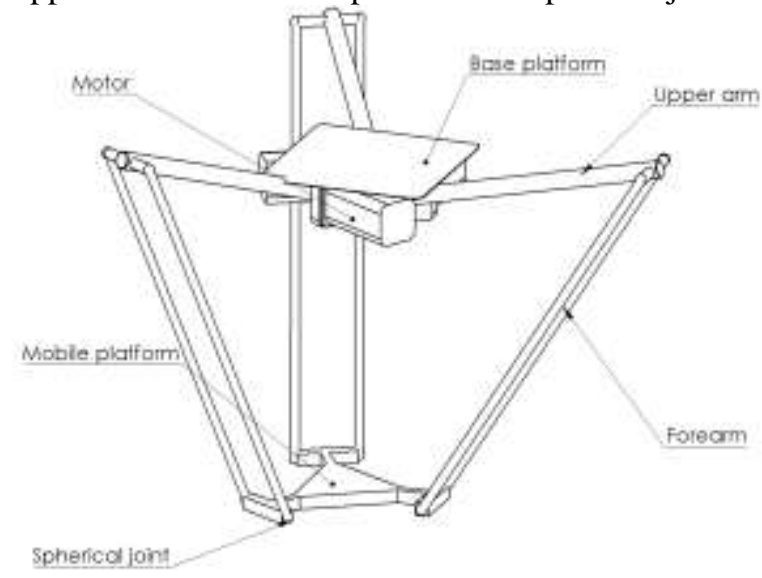

Fig. 1. The Delta-3 robot

In order to establish the kinematic equations, the deferent parameters used are presented in Fig. 2 and described below: 


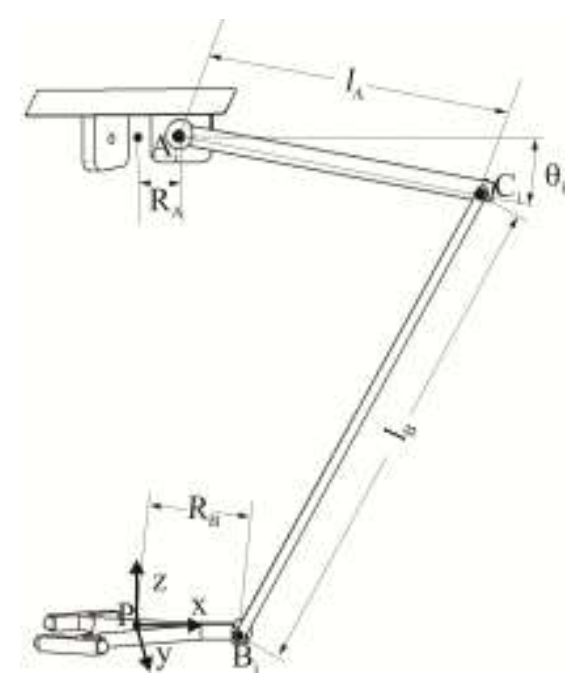

Fig. 2. Parallel kinematic chain of Delta-3 robot

$P \quad$ Mobile platform's center.

$\theta_{i} \quad$ Joint angle of the upper arms.

$\alpha_{i} \quad$ Frame rotation angle for the arm attached to the motor.

$C_{i} \quad$ Joint point between the lower and upper arms

$B_{i} \quad$ Contact point between the mobile platform and the forearm.

$l_{A} \quad$ Length of the upper arm

$l_{B} \quad$ Length of the forearm

$R \quad$ Defined as $R=R_{A}-R_{B}$

$R_{A} \quad$ Distance between the center of the fixed base and the upper arm rotation axis.

$R_{B} \quad$ Distance between the center of the mobile platform and lower extremity of forearm.

\section{Delta-3 Robot KinEMATICS}

This section concerns studding the geometry and the motion of the Delta-3 robot without considering neither masses nor forces.

\section{A. Forward Kinematics}

Forward kinematics refers to the use of the kinematic equations of the robot in order to find the Cartesian coordinates $(x, y, z)$ of the mobile platform's center $P$ from specified values of joint coordinates $\theta_{i}$. For the Delta-3 robot R.Clavel [5] proposed a simplification based on geometry analytical relations. It consists of the intersection of three spheres, each with the center at the elbow $C_{i}$ of the parallel kinematic chains, and the radius $l_{B}$ is the forearms lengths. The intersection of these spheres will result in two points, with one of them is the point $P$.

Thereby to obtain the Cartesian coordinates $(x, y, z)$ of $P$, it is merely to solve the equation (1) of the intersection of the three spheres below:

$$
\begin{aligned}
& x^{2}-2 x\left(R+l_{A} \cos \theta_{i}\right) \cos \alpha_{i}+y^{2}-2 y\left(R+l_{A} \cos \theta_{i}\right) \\
& \sin \alpha_{i}+z^{2}+2 z l_{A} \sin \theta_{i}=l_{B}{ }^{2}-l_{A}{ }^{2}-R^{2}-2 R l_{A} \cos \theta_{i}
\end{aligned}
$$

\section{B. Inverse Kinematics}

The inverse kinematics of the Delta robot defines the joint coordinates $\theta_{i}$ from specified values of the Cartesian coordinates $(x, y, z)$. To obtain it, it is required to resolve (1) according to the joint variables $\theta_{i}$.

\section{Velocity Kinematics}

This relationship demines the velocity coordinates $\dot{X}_{n}$ of the mobile platform's center based on the joint velocities $\dot{\theta}$. To calculate it, it is important to determine the Jacobin matrix $J$. The easiest way to obtain it, is by considering a set of constraint equations linking the operational-space variables to the joint-space variables [6].

The three constraint equations of the Delta-3 robot can be expressed as:

$$
\left\|C_{i} B_{i}\right\|_{2}^{2}-l_{B}^{2}=0 \quad i=1,2,3
$$

Developing (2), we obtain the equation of the velocity kinematics (3).

$$
\dot{X}_{n}=J \dot{\theta}
$$

\section{Acceleration Kinematics}

This relationship describes the acceleration coordinates $\ddot{X}_{n}$ of the mobile platform's center based on joint acceleration coordinates $\ddot{\theta}$. To obtain this model, we just have to derivate by time the velocity kinematics equation (4).

$$
\ddot{X}_{n}=\dot{J} \dot{\theta}+J \ddot{\theta}
$$

\section{DYNAMIC MODEL}

The dynamic model allows to determine the torque/force of the actuators for generating a desired path. For the Delta-3 robot that model can be calculated using different methods (Lagrange, L'Alembert and Newton-Euler). This system is fully investigated by Olsson [7]. The Fig. 3 illustrates the dynamic system which is composed of two subsystem. The first one is considered linear and decoupled since each of the inputs $\tau_{m}$ affects linearly one of the outputs $\theta_{m}$ only. The last one is non-linear and coupled because it includes all the complex relationships between the output $d$ and the inputs $\theta_{m}, \dot{\theta}_{m}$ and $\ddot{\theta}_{m}$ 


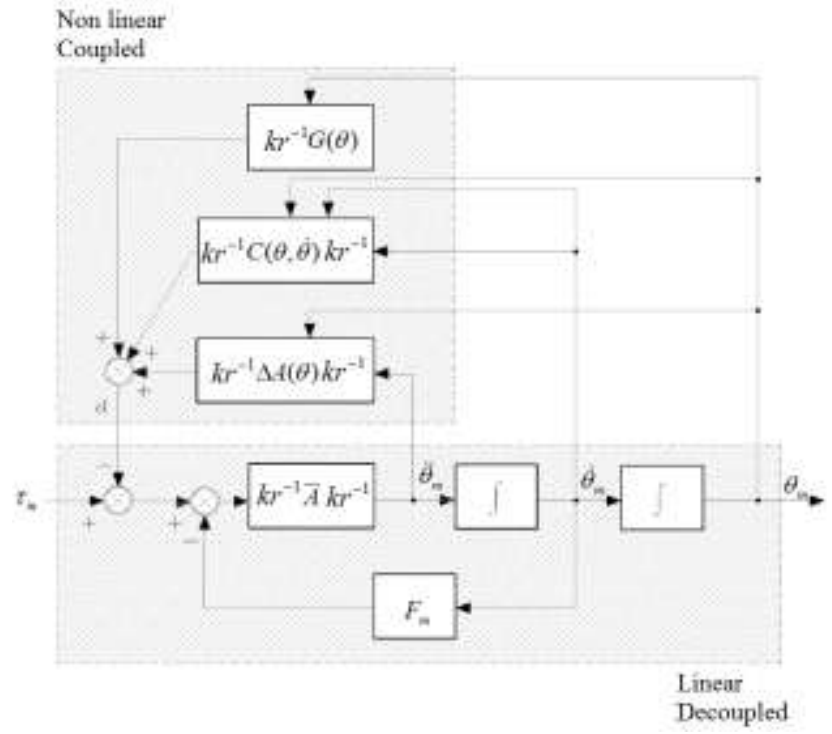

Fig. 3. Block scheme of the Delta-3 robot with drives [8]

$\tau_{m} \quad$ Actuator's shaft torque.

$K_{r} \quad$ Gear reduction ratio.

A Inertia matrix which is formed by constant terms $\bar{A}$ and functions of sine and cosine $\Delta A$.

$\theta_{m} \quad$ Motor's angular variable

$\theta$ Angular position variable of the robot's arm

$C$ Describes the Centrifugal and Coriolis forces values.

$G \quad$ Values of gravitational forces that act on the robot.

\section{CONTROL OF THE SYSTEM}

In this section we present the control techniques and the convenient actuators used to actuated the robot.

\section{A. Motors}

Motors that we have chosen to actuate the three arms of the Delta-3 robot are permanent magnet DC motors (PMDC). The most important advantage that concerns us for that type of electric motors is that the input voltage of the motor is proportional to the output velocity (linearity), which helps us to control the 3 arms of the Delta-3.

\section{B. Correction and Stability}

To choose the control structure of the Delta-3 robot, it should be first noted that the effective rejection of the disturbance $d$ at the output $\theta_{m}$ is ensured by a large gain value of the amplifier before the intervention point of disturbance. The aim of the integral action in the controller is to cancel the effect of the gravitational component on the output at the steady state [8], where the use of a Proportional-Integral (PI) correction is required.

To improve the dynamic performances it is better to control each of the acceleration, speed, and position separately as illustrated in Fig. 4.

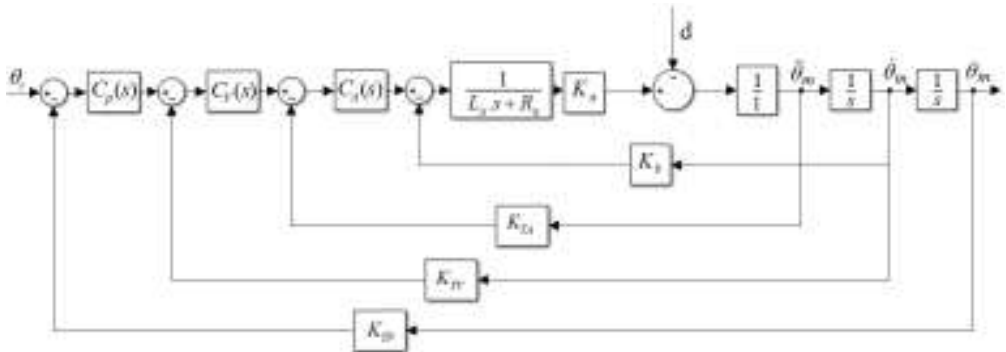

Fig. 4. General block scheme of the control system

With $\theta_{r}$ is the shaft motor's reference input, $K_{a}$ is the motor's torque constant, $K_{b}$ is the motor's velocity constant. $C_{p}(s), C_{V}(s)$ and $C_{A}(s)$ represent respectively the position, velocity and acceleration controllers. In addition, $K_{T P}, K_{T V}$ and $K_{T A}$ are respectively the transaction constants.

The closed loop of the acceleration ensures that within the working interval $\left(6.10^{-4} \mathrm{~Hz}\right.$, to $2.10^{5} \mathrm{~Hz}$ ) the output follows the input regardless the value of the load, which is considered a disturbance, as illustrated in Fig. 5.

We notice that the phase diagram never crosses the threshold of $-180^{\circ}$ which means that the system is stable.
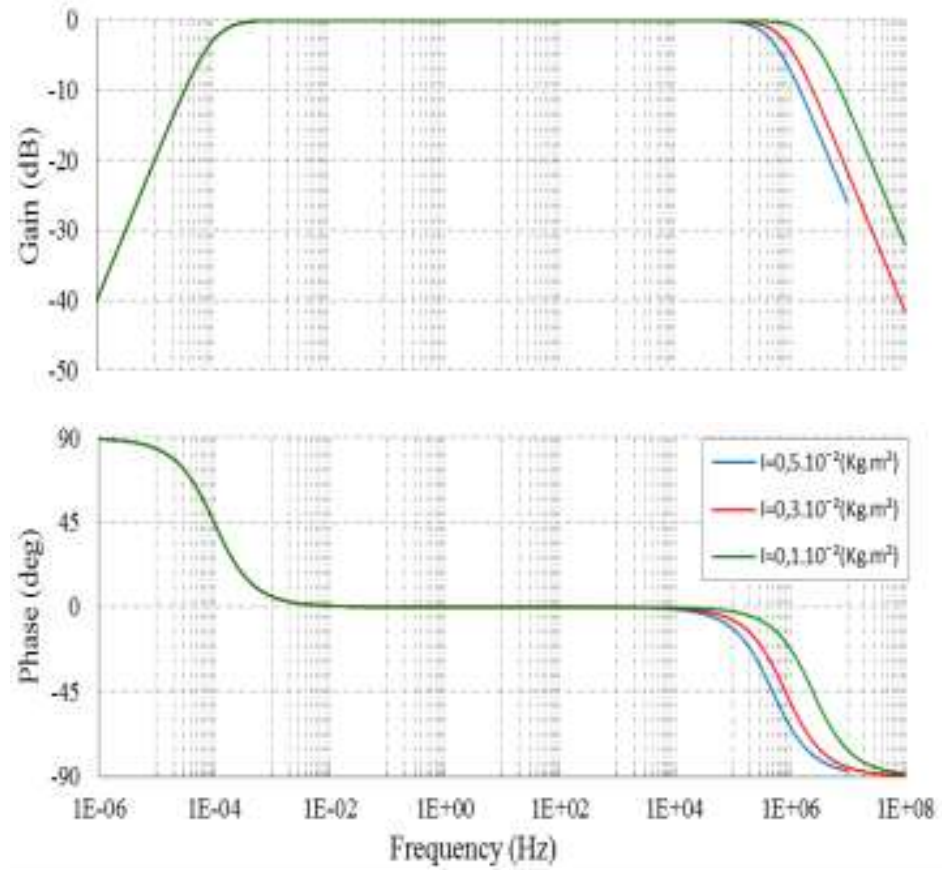

Fig. 5. Bode Diagram of closed loop of the acceleration

In order to test the immunity of the system against disturbances, we applied a sinusoidal disturbance signal and changed the gain $K_{a}$ of the corrector. More the gain is higher, more the sensitivity to the disturbance is lower, as illustrated in Fig. 6. 


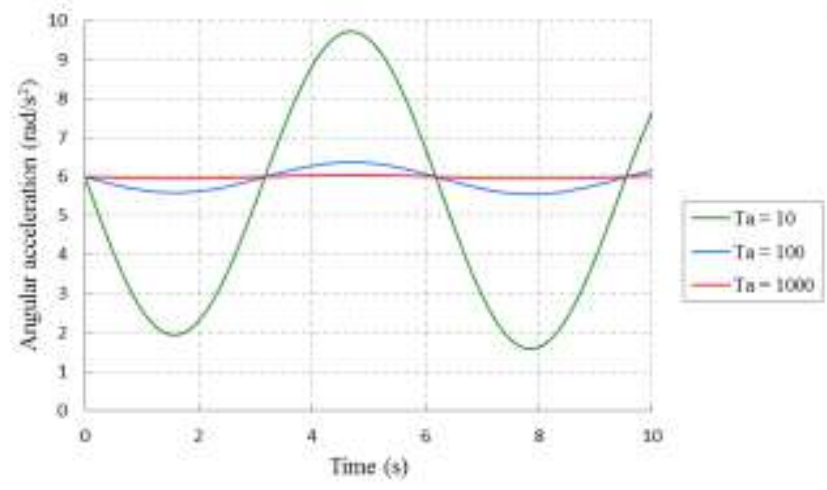

Fig. 6. Sensitivity to disturbances

The closed loop diagram of the velocity (Fig. 7) shows a stable system and bandwidth of $0 \mathrm{~Hz}$ to $100 \mathrm{~Hz}$. We notice that the bandwidth of the acceleration is much larger than of the velocity. This is due to the integral fact that reduces the bandwidth.

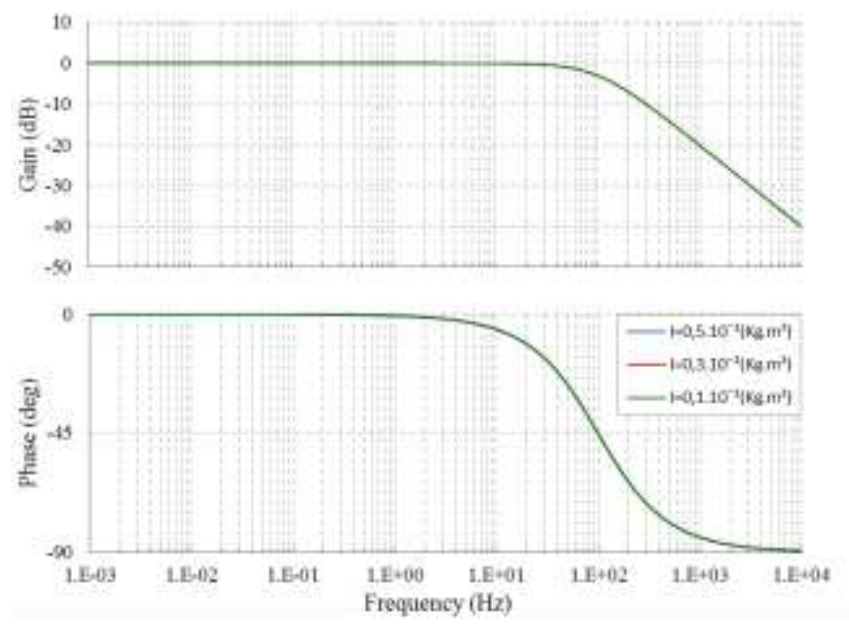

Fig. 7. Bode Diagram of closed loop of the velocity

To determine the characteristics of the closed loop of the position, we drew Bode diagram Fig. 8.
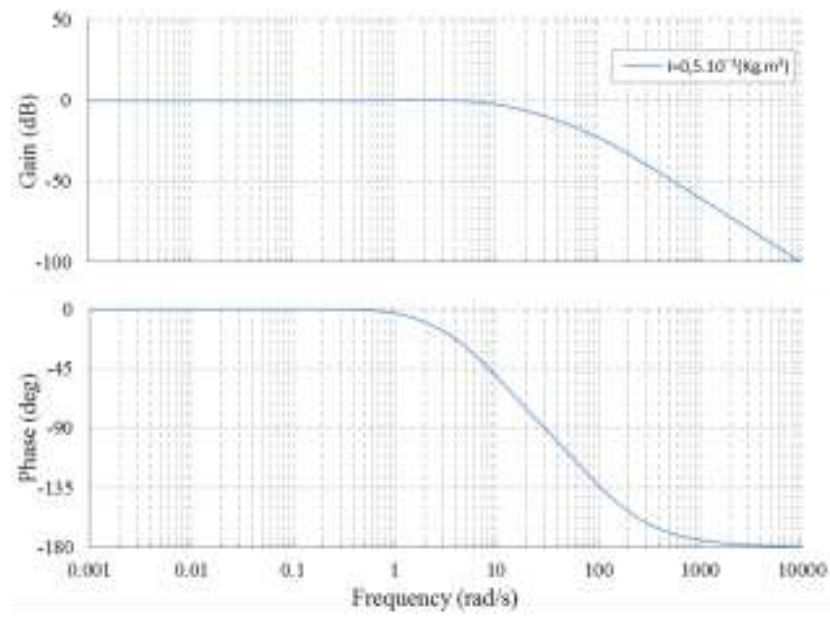

Fig. 8. Bode Diagram of closed loop of the position

The diagram shows that the bandwidth varies from $0 \mathrm{~Hz}$ to $6 \mathrm{~Hz}$. Therefore, in order to get a good response, the spectrum of the input should be within this interval

\section{Modeling AND SIMULATION}

After the collection and the definition of all parameters that describe the Delta-3 robot's behavior, it remains the modeling and simulation. The objective of this section is to create a dynamic model mechanism of the Delta-3 robot, using Solidworks in combination with SimMechanics and Matlab Simulink.

\section{A. Modeling with SolidWorks}

The modeling of delta-3 robot in solidworks was made in two stages:

- Parts Creating: Each part of the Delta-3 robot was built separately. The model includes eight parts: fixed base, mobile plate, three upper arms and three forearms.

- Parts assembling: This is done using Solidworks Assembly. We can build a complex assembly, consisting of several components, which may be parts or assemblies. Adding an element, we could create a link between the assembly and its component [9]. The assembly of the Delta-3 robot is done by the connecting:

- The three upper arm with the fixed base by revolute joints

- The three forearms with the mobile plate by spherical joints.

\section{B. Delta-3 Robot Simulation}

The simulation of the Delta-3 robot is done using Simulink with the combination of SimMechanics. The Simulink model structure of the Delta-3 robot is divided into different parts such as the trajectories generator, the block of the kinematic model and the dynamic of the robot control unit, three motors, and block of 3D simulation. Fig. 14 illustrates the Simulink model of Delta-3 robot, with the position of each block.

The execution process of this model is implemented in several stages. At the beginning we have to select the desired trajectory of the mobile platform's center according to Cartesian coordinates (X, Y, Z), then the inverse kinematics bloc transforms them to the upper arms' joints articulation angles, and these themselves will be convert to actuator's shafts angles using $K_{r}$ which is a reduction element. Finally To move each of the Delta-3 robot's arms, the control unit bloc takes the shafts angle' vector of the motors as an input, then calculates the necessary current values for the motor to reach the desire trajectory. In order to get a 3 Dimensional (3D) simulation of Delta-3 robot, the SolidWork CAD model is converted to a SimMechanics model which is represented by 3D simulation Fig. 15 Based on the output vector that describes the reel motors shafts angle' position, this bloc generates a 3D animation that visualizes the motion of the robot. In addition to that, using forward kinematics \& scope blocs we get the graphical data which describes the reel path of the mobile platform's center according to the Cartesian coordinates.

To test the Simulink model of the Delta-3 robot, we applied a spiral trajectory (Fig. 9) on the XY plan with $\mathrm{Z}=22 \mathrm{~mm}$. We have recorded the position, velocity and the acceleration. 


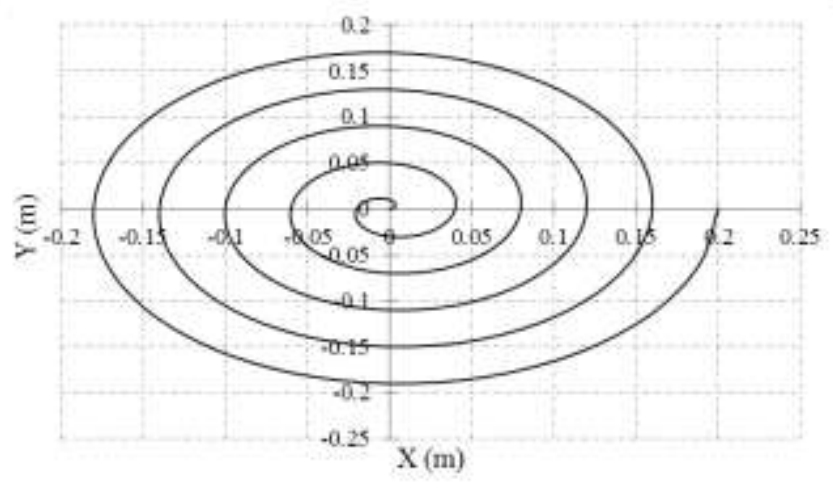

The results are illustrated in Fig. 9, Fig. 10, Fig. 11 and Fig. 12

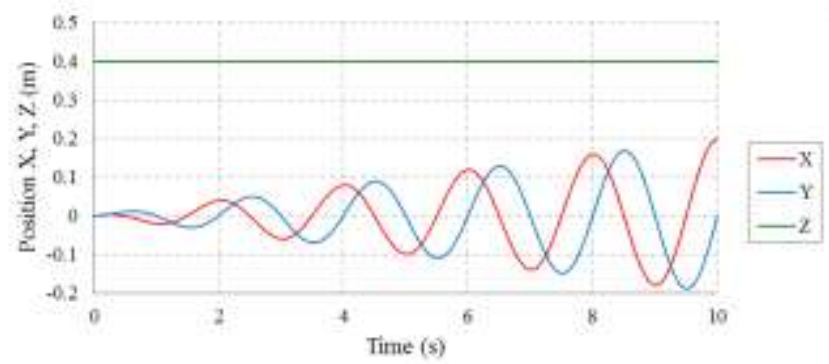

Fig. 9. Setpoint of the time position, of the mobile platform's center (Cartesian coordinates)

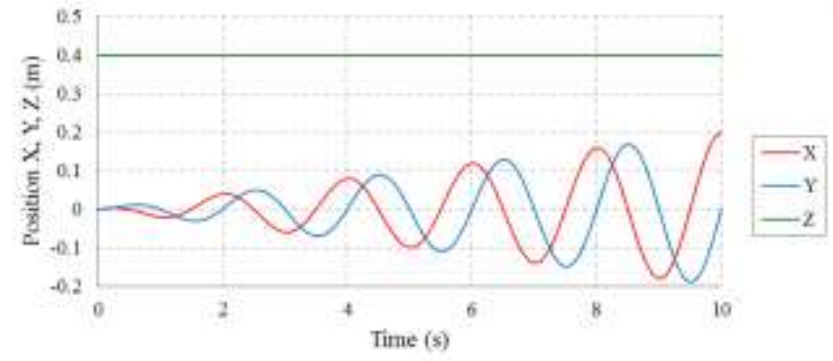

Fig. 10. Time position obtained of the mobile platform's center according to the Cartesian coordinates
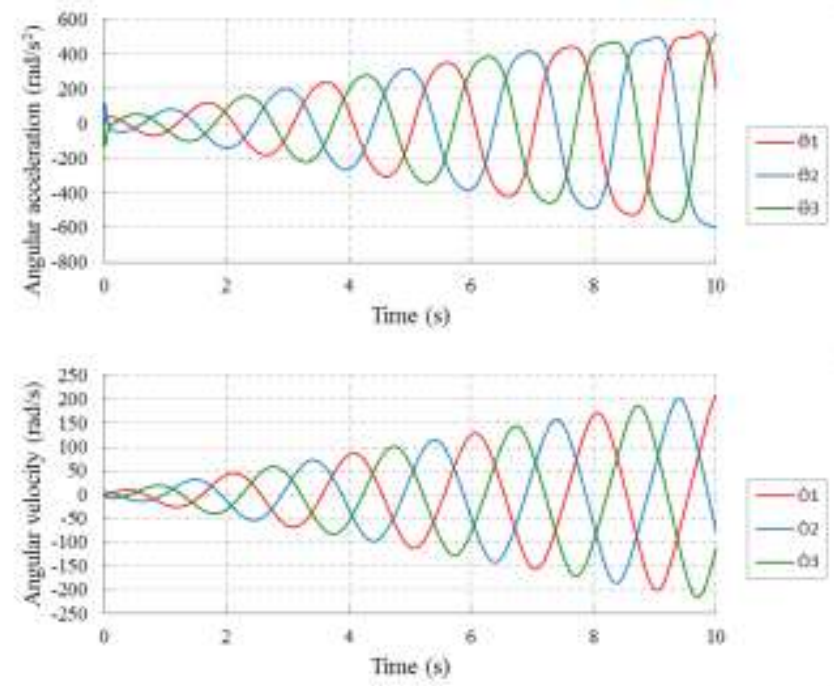

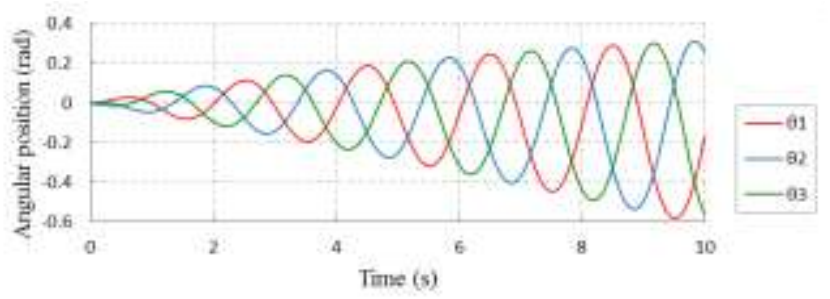

Fig. 11. Setpoint of angular acceleration, velocity and position of the three motors
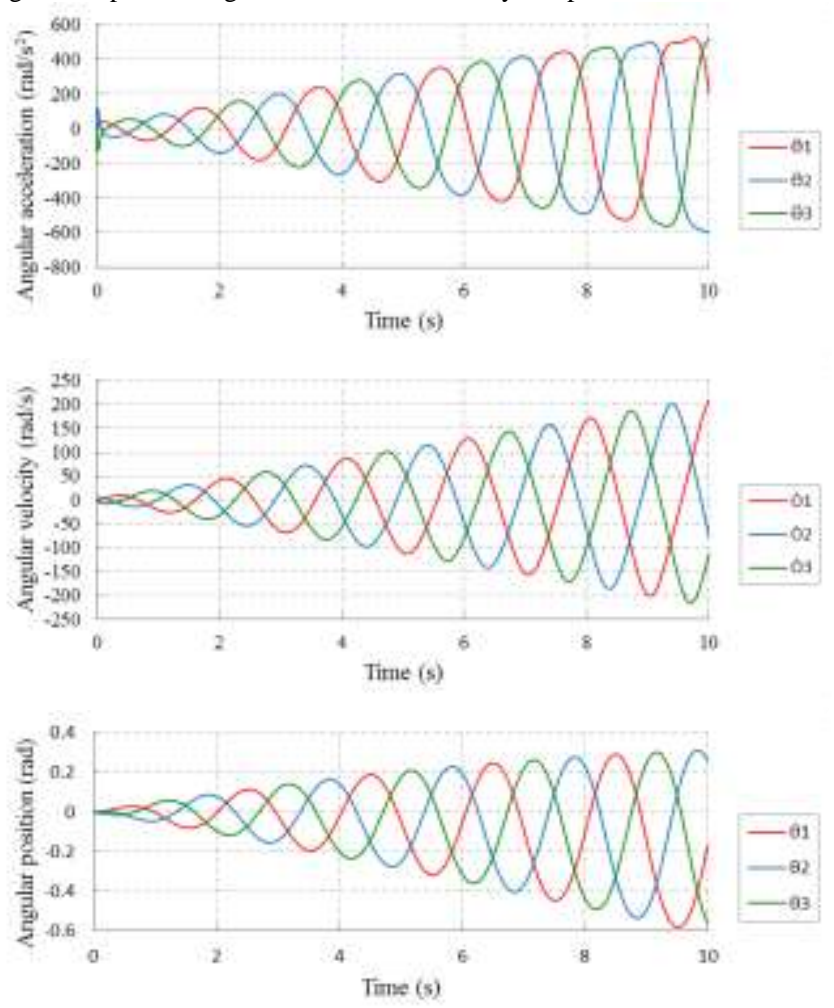

Fig. 12. Result angular acceleration, velocity and position of the motors

The obtained results show that our system has followed the setpoint, with good spatial and temporal precision.

\section{CONCLUSION}

The kinematics study is absolutely essential for programing robot's operational space. While dynamic model allows us to define forces and velocities necessary to define the specifications of the motors to use. The study of the stability helps us to define the domain of the spectrum where system shows better performances. It allowed us to decouple the three arms of the robot, and getting high precision in position. This is done thanks to inner closed loop around acceleration, velocity, and position. The right choice of the parameters allows to minimize the responding time and the overshoot.

\section{REFERENCES}

[1] CDC, "CDC estimates 1 in 68 children has been identified with autism spectrum disorder," 27 March 2014. [Online]. Available: http://www.cdc.gov/media/releases/2014/p0327-autism-spectrum -disorder.html.

[2] F. Petric, K. Hrvatinić, A. Babić, L. Malovan, D. Miklić, Z. Kovačić, M. Cepanec, J. Stošić and S. Šimleša, "Four Tasks of a Robot-assisted Autism 
Spectrum Disorder Diagnostic Protocol: First Clinical Tests," in Global Humanitarian Technology Conference (GHTC)., San Jose, 2014, pp 1-8 https://doi.org/10.1109/GHTC.2014.6970331

[3] R. Clavel, "Dispositive pour le deplacement et le positionnement d'un element dans l'espace," Patent Wo8703528a1, Jun 1987.

[4] I. Bonev, "Delta Parallel Robot - the Story of Success," 2003. [Online]. Available: http://www.parallemic.org/Reviews/Review002.html.

[5] R. Clavel, "Conception d'un robot parallèle rapide à 4 degrés de liberté," $\mathrm{Ph}$.D. dissertation, Dep. Mechatronics. Ecole Polytechnique fédérale de Lausanne, Swiss, 1991

[6] A. Codourey, "Dynamic Modeling of Parallel Robots for Computed-Torque Control Implementation," The International Journal of Robotics Research, vol. 17, no. 12, pp. 1325-1336, Dec 1998. https://doi.org/10.1177/027836499801701205

[7] A. Olsson, "Modeling and control of a Delta-3 robot," M.S. thesis, Dept. Automatic Control., Lund University, Sweden, 2009.

[8] L. Sciavicco and B. Siciliano, Modelling and Control of Robot Manipulators, 2nd ed., London, U.K.: Springer-Verlag, 2005, ch. 6, pp. 213-268.

[9] S. S. Duicu and L. Popa, "Modeling and simulation of the 5-axis robot arm trajectory," International Conference on Applied Informatics and Communications, pp. 154-159, Greece: WSEAS Press, 2012. 